\title{
AVALIAÇÃO DOS NÍVEIS SÉRICOS DE VANCOMICINA EM PACIENTES INTERNADOS EM UM HOSPITAL DE ENSINO DA REGIÃO CENTRAL DO RIO GRANDE DO SUL - BRASIL
}

\author{
Monica Basso Zanotto ${ }^{1}$ \\ Patrícia Raquel Wappler ${ }^{2}$ \\ Pedro Alves d'Azevedo ${ }^{3}$ \\ Marcelo Carneiro ${ }^{4}$ \\ Janine de Melo Rauber ${ }^{5}$ \\ Andreia Rosane de Moura Valim ${ }^{6}$
}

\begin{abstract}
RESUMO
A vancomicina é um antibiótico glicopeptídeo efetivo em infecções graves por bactérias gram-positivas. Necessita atingir um nível sérico adequado para alcançar efetividade terapêutica e requer monitorização constante durante seu uso uma vez que pode causar toxicidade. $\mathrm{O}$ objetivo deste trabalho foi verificar qual o nível sérico de vancomicina nos pacientes internados em um hospital de ensino da região central do Rio Grande do Sul. Este estudo foi realizado com 69 pacientes, sendo 22 recém-nascidos, no período de julho de 2012 a dezembro 2013. As dosagens séricas de vancomicina foram realizadas após 72 horas do início do tratamento com o antimicrobiano, 30 minutos antes de sua administração através de imunoensaio de micropartículas por quimioluminescência. Foram realizadas e analisadas 117 dosagens séricas de vancomicina, sendo que apenas $19,4 \%$ das dosagens permaneceram na faixa ideal $(10-20 \mu \mathrm{g} / \mathrm{mL})$, enquanto que $38,1 \%$ permaneceram abaixo e $42,5 \%$ acima da faixa adequada. Com base nestes resultados, concluímos que é importante mensurar os níveis séricos deste antimicrobiano, a fim de evitar a toxicidade e alcançar níveis adequados para o sucesso terapêutico.
\end{abstract}

Palavras-chave: Vancomicina. Monitoramento de medicamentos. Bactérias gram-positivas. Infecção hospitalar

\footnotetext{
ABSTRACT

Vancomycin is a glycopeptide antibiotic effective for gram-positive severe infections. To therapeutic effectiveness, it is necessary achieve appropriated serum levels and it requires constant monitoring since it can cause toxicity. The aim of this study was to verify the level of vancomycin serum in patients admitted to a teaching hospital in the central region of Rio Grande do Sul. This study was conducted with 69 patients, where 22 were newborns, in the period July 2012 to December 2013. Vancomycin serum levels were performed 72 hours after initiation of treatment with antimicrobial, 30 minutes before their administration, by

${ }^{1}$ Acadêmica do Curso de Medicina da Universidade de Santa Cruz do Sul - UNISC. 〈bzmoni@ gmail.com>

${ }^{2}$ Acadêmica do Curso de Farmácia da Universidade de Santa Cruz do Sul - UNISC. 〈pathyw@ hotmail.com>

${ }^{3}$ Professor-Pesquisador do Programa de Pós-Graduação em Ciências da Saúde da Universidade Federal de Ciências da Saúde de Porto Alegre - UFCSPA. <pedro_dazevedo@yahoo.com.br>

${ }^{4}$ Professor do Departamento de Biologia e Farmácia da Universidade de Santa Cruz do Sul - UNISC. <carneiromarcelo@yahoo.com.br>

${ }^{5}$ Farmacêutica e Doutoranda em Ciências da Saúde pela Universidade Federal de Ciências da Saúde de Porto Alegre - UFCSPA. <janine.farm@gmail.com>

${ }^{6}$ Professora e Pesquisadora do Departamento de Biologia e Farmácia e do Programa de Pós-Graduação em Promoção de Saúde na Universidade de Santa Cruz do Sul - UNISC. 〈avalim@unisc.br>
} 
chemiluminescent microparticle immunoassay. 117 serum vancomycin were performed and analyzed, with only $19.5 \%$ of the dose remained in the ideal range $(10-20 \mu \mathrm{g} / \mathrm{mL})$, whereas $38.1 \%$ were below and $42.5 \%$ above the recommended range. Based on these results, we conclude that it is important to measure the serum levels of this antibiotic in order to avoid toxicity and achieve adequate success therapeutic.

Keywords: Vancomycin. Drug monitoring. Gram-positive bacteria. Nosocomial infections.

\section{INTRODUÇÃO}

A vancomicina é um antimicrobiano muito efetivo em infecções graves por bactérias gram-positivas. Alguns microrganismos gram-positivos, como Staphylococcus aureus, Enterococcus faecalis e Enterococcus faecium, são considerados patógenos oportunistas em pacientes hospitalizados e costumam apresentar resistência a diversos antimicrobianos, fato que compromete a terapia (WILLEMS et al., 2011).

Nas últimas décadas, um importante problema de saúde pública tem causado alerta: o aumento da incidência de resistência microbiana aliado ao surgimento de bactérias multirresistentes (UEKOTTER et al., 2011). Neste sentido, o tratamento de infecções causadas por $S$. aureus tem sido dificultado pela grande prevalência de cepas resistentes à meticilina/oxacilina (MRSA) (CALFEE, 2013).

Em casos de infecções por MRSA, o antimicrobiano de primeira escolha para o tratamento é a vancomicina, medicamento da classe dos glicopeptídeos (AVENT et al., 2013). Trata-se de uma opção bastante eficiente, uma vez que é utilizada desde a década de 1950 e são poucos os relatos de resistência (VRSA). No entanto, ainda que a maioria dos isolados seja sensível, são encontrados muitos registros de falha na terapia com vancomicina em estudos publicados desde os anos 1970 (KOLLEF, 2007).

Alguns autores demonstraram que fatores clínicos, como concentração sérica do antibiótico reduzida (inferior a $15 \mu \mathrm{g} / \mathrm{mL}$ ), tratamento prévio com vancomicina, presença de outras co-morbidades e demais características do paciente influenciam no desfecho clínico (PRICE et al., 2010; KULLAR et al., 2011; BROWN et al., 2011).

Segundo o consenso da American Society of Health-System Pharmacist, Infectious Disease Society of America e Society of Infection Disease Pharmacists (RYBAK et al., 2009), a vancomicina necessita atingir níveis mínimos séricos adequados $(10-20 \mu \mathrm{g} / \mathrm{mL})$ para alcançar efetividade terapêutica e evitar a toxicidade. Além disso, a monitorização do antimicrobiano é justificável devido à complexidade de sua farmacocinética, fato que afeta 
significativamente o sucesso terapêutico (REIS; GRISI, 1996). Neste contexto, o presente trabalho tem o objetivo de avaliar os níveis séricos de vancomicina em pacientes internados, tanto em Unidades de Terapia Intensiva quanto em enfermarias, em um hospital de ensino da região central do Rio Grande do Sul.

\section{FUNDAMENTAÇÃO TEÓRICA}

Infecções causadas por bactérias gram-positivas são frequentes tanto na comunidade quanto em pacientes hospitalizados (WILLEMS et al., 2011). S. aureus, principal representante dos microrganismos gram-positivos, é componente da microbiota normal e está presente na pele e nas narinas de aproximadamente um terço da população (PEACOCKET et al., 2001; NULENS et al., 2005; GORWITZ et al., 2008). E. faecalis e E. faecium também fazem parte da microbiota do trato gastrintestinal humano (BOOIJINK et al., 2010) e, assim como S. aureus, são típicos patógenos oportunistas que causam diversas infecções (WILLEMS et al., 2011).

A importância clínica dos enterococos evidenciou-se a partir de 1980, quando foram registrados altos índices de resistência à ampicilina, principal droga de escolha para o tratamento até o momento. Em 1988 foram publicados os primeiros casos de resistência de enterococos à vancomicina (VRE), alternativa importante no caso de resistência à ampicilina (LECLERCQ et al., 1988; UTTLEY et al., 1988).

Segundo Sievert e colaboradores (2013), o gênero Enterococcus spp. permanece entre os principais patógenos causadores de infecções hospitalares nos Estados Unidos, sendo que em 2009 e 2010 foi responsável por 14\% destas infecções. Além disso, foram encontrados diferentes índices de resistência à vancomicina entre as espécies, detectando-se de 7-10\% de resistência para E. faecalis e de $80-85 \%$ para E. faecium.

No caso do $S$. aureus, há descrição de diferentes formas de resistência a antibióticos, sendo que o primeiro relato surgiu na década de 40, com a introdução da penicilina. Esse tipo de resistência era mediado por genes que codificavam enzimas chamadas $\beta$-lactamases, responsáveis pela degradação do antibiótico. No início da década de 60, a meticilina foi introduzida como alternativa terapêutica para as bactérias produtoras de $\beta$-lactamases, uma vez que essa droga não sofria a ação destas enzimas. No entanto, no ano seguinte, foram encontrados os primeiros isolados resistentes à meticilina (MRSA) e sua prevalência tem sido considerada um grande problema de saúde pública atualmente (UEKOTTER et al., 2011). 
O gênero Staphylococcus spp. foi responsável por 27\% das infecções hospitalares nos Estados Unidos entre os anos de 2009 e 2010 (SIEVERT et al., 2013). A espécie S. aureus foi reportada em $16 \%$ das infecções hospitalares deste país e a prevalência de MRSA foi de 46 a $63 \%$ entre os isolados. Rosenthal e colaboradores (2012), em estudo realizado de 2004 a 2009 em 36 países da América Latina, Ásia, África e Europa pelo International Nosocomial Infection Control Consortium (INICC), registraram entre 71 e $84 \%$ de resistência à meticilina para S. aureus.

A vancomicina é a primeira escolha para o tratamento intravenoso de MRSA, bem como para infecções graves por cocos gram-positivos no caso de pacientes hospitalizados. Em ensaios clínicos, antimicrobianos lançados mais recentemente, como a daptomicina, linezolida e tigeciclina, não superam a atividade da vancomicina, medicamento desenvolvido e comercializado há mais de 50 anos (DELEO et al., 2010).

A vancomicina age inibindo a síntese da parede celular bacteriana, impedindo o acoplamento dos polímeros de peptidoglicano. A resistência ao antimicrobiano ocorre por meio de uma modificação no peptídeo da parede celular do microrganismo. Assim, há alteração do fragmento de D-alanil-D-alanina, tanto para D-alanil-D-lactato quanto para Dalanil-D-serina, impossibilitando a ligação do fármaco. Esta resistência é mediada por loci gênicos, sendo que o mais frequente é o gene vanA, carreado por plasmídeos e transposon, que conferem resistência ao antibiótico (PÉRICHON, COUVARLIN, 2004; LEVINE, 2006).

Em 1997, Hiramatsu e colaboradores (1997) descreveram no Japão um isolado de $S$. aureus com susceptibilidade reduzida à vancomicina (VISA). Após esta notificação, este fenótipo foi relatado também em outros locais, como Estados Unidos, Austrália, França, Coréia do Sul, Israel, África do Sul, entre outros países, inclusive o Brasil (OLIVEIRA et al., 2001).

A resistência completa do $S$. aureus ao glicopeptídeo (VRSA) foi descrita primeiramente nos Estados Unidos em 2002 pelo Centers for Disease Control and Prevention (CDC, 2002), sendo que até o momento foram notificados apenas 33 isolados com esta importante resistência (ASKARI et al., 2013). O relato da primeira cepa de S. aureus resistente à vancomicina (VRSA) destaca que a aquisição do gene vanA pode ter ocorrido a partir de E. faecalis resistente à vancomicina (VRE) que também foi isolado no paciente naquela ocasião (CDC, 2002).

No caso de isolados VISA, percebe-se o aumento da MIC para o glicopeptídeo através de métodos fenotípicos, porém não é detectado o gene van, bem como seus subtipos, por meio de técnicas moleculares. O mecanismo de resistência intermediária parece estar associado a 
um espessamento da parede celular da bactéria, que dificulta a ação da vancomicina (HOWDEN et al., 2010).

Segundo o Clinical and Laboratory Standards Institute (CLSI), a sensibilidade à vancomicina é inferida através da MIC. De acordo com critérios adotados a partir de 2006 para S. aureus (CLSI, 2013), os isolados com MIC menor ou igual a $2 \mu \mathrm{g} / \mathrm{mL}$ são considerados sensíveis à vancomicina; as cepas de MIC entre 4 e $8 \mu \mathrm{g} / \mathrm{mL}$ são ditas de sensibilidade intermediária (VISA); e culturas com MIC maior do que $16 \mu \mathrm{g} / \mathrm{mL}$ são resistentes ao antimicrobiano (VRSA).

Embora estas categorias sejam amplamente conhecidas na microbiologia (sensível, intermediário e resistente), atualmente para $S$. aureus está sendo definida uma nova classificação: a hetero-resistência intermediária à vancomicina (hVISA). Essencialmente, um isolado hVISA é um isolado de S. aureus com MIC para vancomicina dentro da faixa suscetível quando avaliado pelo métodos convencionais de rotina, mas neste isolado há uma proporção da população de células (1:1.000.000) que está na faixa de sensibilidade intermediária (HIRAMATSU, 2001). Estima-se que aproximadamente 60\% dos isolados de MRSA com MIC de $2 \mu \mathrm{g} / \mathrm{mL}$ são cepas de hVISA (HOWDEN et al., 2010).

De fato, a vancomicina continua sendo o tratamento padrão para infecções causadas por MRSA. No entanto, o surgimento de isolados hVISA tem comprometido a utilização deste antibiótico, devido a taxas significativas de falhas no desfecho clínico do paciente nestes casos (VAN HAL, PATERSON, 2011; CASAPAO et al., 2013). Além da conhecida toxicidade deste antimicrobiano, falhas no tratamento de endocardites, bacteremias e pneumonias por cepas de S. aureus sensíveis e resistentes à meticilina (MSSA e MRSA) estão sendo relatadas desde 1977 até o momento (KOLLEF, 2007).

Diversos estudos relatam dificuldades no tratamento de MRSA, normalmente em casos de isolados com altos valores de concentração mínima inibitória (MIC) para vancomicina (SORIANO et al., 2008; LODISE et al., 2008; YOON et al., 2010). Assim, a ineficácia é atribuída à menor proporção de área sob a curva (AUC) sobre a MIC, sendo esta imprescindível para o sucesso da terapia com vancomicina (HOLLAND, FOWLER, 2011). Entretanto, há controvérsia a respeito da real influência da MIC no desfecho da terapia com vancomicina.

Devido ao fenômeno de aumento crescente da MIC, recomenda-se que a concentração sérica mínima de vancomicina deve ser mantida, pelo menos, acima de $10 \mu \mathrm{g} / \mathrm{mL}$ para as infecções de menor gravidade - como, por exemplo, celulite - e entre 15 e $20 \mu \mathrm{g} / \mathrm{mL}$ para as 
infecções mais graves, tais como meningite, bacteremia, osteomielite, endocardite e pneumonia (RYBAK et al., 2009; BROOME; SO, 2011).

Sabe-se que algumas situações especiais, como pacientes gravemente enfermos, dialíticos, obesos e crianças requerem dosagens diferenciadas do antimicrobiano e ajuste de dose conforme avaliação dos níveis séricos de vancomicina (AVENT et al., 2013). Nos pacientes obesos, estudos evidenciam que há alteração da farmacocinética da vancomicina, tanto no volume de distribuição, devido ao excesso de tecido adiposo e proteínas de ligação nestes pacientes, quanto na eliminação renal. Ambos os parâmetros farmacocinéticos influenciam diretamente na dose necessária para atingir níveis terapêuticos, bem como no intervalo entre doses para manutenção da concentração adequada ao longo do tratamento (GRACE; GOODBAR; FOUSHEE, 2012).

Com relação aos pacientes com doença renal em estágio final e em processo dialítico, o fato do antibiótico ser eliminado exclusivamente por via renal aumenta significativamente as chances da vancomicina atingir níveis tóxicos na circulação sanguínea. O tempo de meiavida de eliminação pode se alterar de 6 a 12 horas (função renal normal) para 100 a 200 horas (VANDECASTEELE; DE BACQUER; DE VRIESE, 2011).

Conforme as orientações práticas da Infectious Disease Society of America para tratamento de infecções por MRSA (LIU et al., 2011), atualmente são recomendadas doses de $15 \mathrm{mg} / \mathrm{kg}$ a cada 6 horas $(60 \mathrm{mg} / \mathrm{kg} / \mathrm{dia})$ para paciente pediátricos com infecções complicadas de pele e tecidos moles, bacteremias, pneumonias, meningites e sepse.

A monitorização da vancocinemia se faz necessária uma vez que níveis baixos do medicamento podem favorecer o surgimento de cepas resistentes de $S$. aureus enquanto que níveis elevados favorecem o surgimento de toxicidade (JAMES; GURK-TUNER, 2001; RYBAK et al., 2009; ELYASI et al., 2012; VAN HAL; PATERSON; LODISE, 2013; YE; TANG; ZHAI, 2013).

\section{MATERIAIS E MÉTODOS}

Foram incluídos no estudo os pacientes internados em um hospital de ensino da região central do Rio Grande do Sul em uso da medicação vancomicina. A monitorização do uso deste glicopeptídeo na instituição teve duração de 18 meses (julho/2012 a dezembro/2013). O presente trabalho foi realizado após aprovação do Comitê de Ética em Pesquisa (CEP) da Universidade Federal de Ciências da Saúde de Porto Alegre e encontra-se protocolado sob nº. 
957/12 (parecer de aprovação $n^{\circ}$. 1779/12). Os pacientes que não apresentavam assinatura no termo de consentimento livre e esclarecido (TCLE) foram excluídos do estudo.

As coletas foram realizadas após 72 horas do início do tratamento com o antimicrobiano, 30 minutos antes de sua administração (concentração no período de vale). Após foram realizadas coletas em intervalo de 5 dias, observando-se os mesmos critérios. Utilizou-se, para as dosagens, imunoensaio de micropartículas por quimioluminescência, realizado por meio do equipamento da Abbott (Architect 2000). Para cada dosagem foi utilizado $1 \mathrm{~mL}$ de sangue. As concentrações séricas no período de vale foram consideradas apropriadas quando permaneceram entre $10-20 \mu \mathrm{g} / \mathrm{mL}$.

Os prontuários dos pacientes também foram analisados a fim de identificar o perfil epidemiológico dos usuários de vancomicina. Foram coletados dados referentes à idade, sexo e desfecho clínico. Para a análise dos resultados utilizou-se o software SPSS versão 19.0, sendo realizados os testes estatísticos Qui-quadrado, Fisher e t-Student, fixando-se em 5\% ( $\mathrm{p}<0,05)$ o nível de significância estatística.

\section{RESULTADOS E DISCUSSÃO}

Neste estudo, foram incluídos 69 pacientes, sendo 29,8\% recém-nascidos, $16 \%$ crianças e 54,2\% adultos, havendo uma prevalência de pacientes do sexo masculino (60,6\%). Em outros estudos realizados anteriormente que avaliaram os níveis séricos de vancomicina, também houve predominância de pacientes do sexo masculino, fato que facilita a comparação entre dados (PEA et al., 2009; DE WAELE et al., 2013). No entanto, Pea (2009) e De Waele (2013) realizaram acompanhamento, respectivamente, de 133 e 221 pacientes já em idade adulta.

No período do estudo, foram realizadas e analisadas 117 dosagens séricas de vancomicina, totalizando uma média de 1,69 dosagens por paciente. A concentração sérica média do antibiótico foi de $20,12 \mu \mathrm{g} / \mathrm{mL}$, sendo a concentração mínima encontrada de 0,24 $\mu \mathrm{g} / \mathrm{mL}$ e a máxima de $65,03 \mu \mathrm{g} / \mathrm{mL}$. Embora a média de concentração esteja muito próxima em relação à faixa ideal recomendada $(10-20 \mu \mathrm{g} / \mathrm{mL})$, foram encontrados vários resultados fora do intervalo adequado.

A distribuição e a frequência das dosagens séricas quantificadas durante o estudo podem ser visualizadas na Figura 1. Semelhante ao encontrado por Alves e colaboradores (2012), a maioria dos pacientes permaneceu com níveis terapêuticos inadequados para o 
medicamento. Este autor analisou os níveis séricos de 38 pacientes, realizando 76 dosagens no período de vale, sendo que apenas $21,1 \%$ dos pacientes permaneceram com níveis apropriados. Dos 85 pacientes analisados no estudo de Almeida (2011), 20\% das dosagens permaneceram abaixo do ideal, enquanto que apenas $23 \%$ foram consideradas adequadas.

\section{Figura 1 - Adequação dos níveis séricos de vancomicina dos 69 pacientes conforme a}

\section{faixa de concentração $(n=117$ dosagens)}

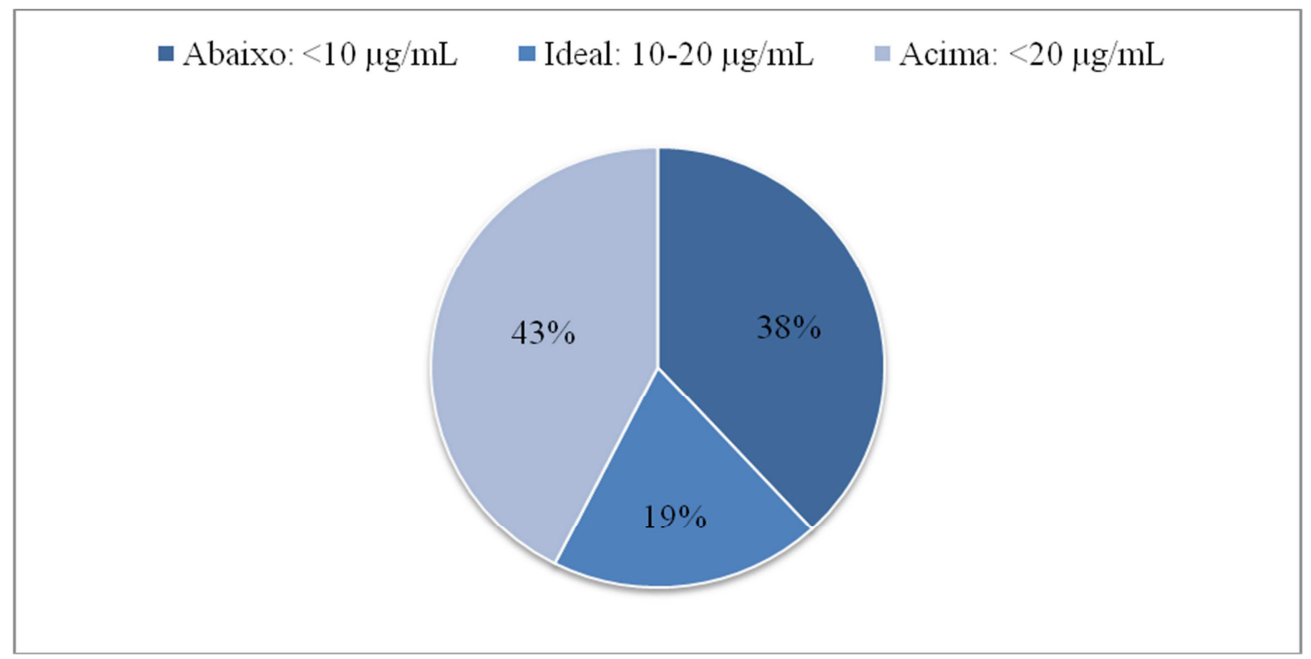

A dose mais frequentemente administrada de vancomicina foi de $1 \mathrm{~g}(32,9 \%)$ para os adultos e de $15 \mathrm{mg} / \mathrm{kg}$ (24,3\%) para as crianças e neonatos. Kim e colaboradores (2010) empregaram doses iniciais de vancomicina entre 40 e $60 \mathrm{mg} / \mathrm{kg} / \mathrm{dia}$ para crianças (de 1 a 16 anos), a fim de verificar qual a melhor dose inicial para atingir níveis apropriados do antibiótico. Estes pesquisadores perceberam que as doses iniciais de $40 \mathrm{mg} / \mathrm{kg} / \mathrm{dia}$ não eram suficientemente altas para alcançar a concentração mínima de $10 \mu \mathrm{g} / \mathrm{mL}$ nos pacientes pediátricos.

A frequência de administração do antibiótico mais prevalente no estudo foi de $12 / 12 \mathrm{~h}$ (30,6\%), seguida de 6/6h (27,8\%) e de 8/8h (22,2\%). De acordo com o consenso da American Society of Health-System Pharmacist, Infectious Disease Society of America e Society of Infection Disease Pharmacists, a frequência de 8 a 12 horas é a recomendada a fim de se atingir uma concentração sérica ótima para a maioria dos pacientes com função renal preservada (RYBAK et al., 2009).

A média das dosagens da vancomicina sérica teve uma diferença significativa quando comparada por sexo (Tabela 1), bem como no estudo de De Waele e colaboradores (2013) 
que o sexo pode influenciar nos níveis de vancomicina sérica, sendo que pacientes do sexo feminino apresentavam maior chance de obter níveis adequados do antibiótico.

Tabela 1 - Concentração sérica média de vancomicina de acordo com variáveis demográficas dos pacientes em geral e conforme características de desfecho em neonatos e crianças

\begin{tabular}{lllrl}
\hline \multicolumn{2}{l}{ Variáveis do estudo } & \multicolumn{3}{c}{ Concentração (média) } \\
\hline $\begin{array}{lllll}\text { Demográficas } \\
(\mathrm{n}=69)\end{array}$ & Sexo & Feminino & $15,56 \mu \mathrm{g} / \mathrm{mL}$ & \\
& & Masculino & $22,67 \mu \mathrm{g} / \mathrm{mL}$ & $\mathrm{p}=0,018$ \\
\cline { 2 - 5 } & Idade & Neonatos e Crianças & $14,48 \mu \mathrm{g} / \mathrm{mL}$ & $\mathrm{p}=0,001$ \\
& & Adultos & $24,95 \mu \mathrm{g} / \mathrm{mL}$ & \\
\hline Desfechos & Piora clínica & Sim & $9,95 \mu \mathrm{g} / \mathrm{mL}$ & $\mathrm{p}=0,156$ \\
$(\mathrm{n}=27)$ & & Não & $16,82 \mu \mathrm{g} / \mathrm{mL}$ & \\
& & Sim & $14,23 \mu \mathrm{g} / \mathrm{mL}$ & \multirow{2}{*}{$\mathrm{p}=0,826$} \\
& Óbito & Não & $15,21 \mu \mathrm{g} / \mathrm{mL}$ & \\
& & &
\end{tabular}

Além disso, conforme a Tabela 1, verificou-se que a concentração sérica de vancomicina nas crianças foi significativamente menor daquela verificada nos adultos. Não foram encontrados estudos comparando dosagens entre adultos e crianças, mas acredita-se que o nível manteve-se mais apropriado entre crianças porque nestes casos é rotina do prescritor ajustar a dose em função do peso do paciente $(\mathrm{mg} / \mathrm{kg})$, fato raro em adultos para os quais são prescritas doses-padrão do antibiótico (por exemplo, 1 g de 12/12 horas).

Avaliando-se apenas o grupo das crianças e recém-nascidos $(n=27)$, verificou-se que $55,2 \%$ destes pacientes apresentaram piora clínica durante o tratamento, sendo que a média de vancomicina sérica foi inferior se comparada aos casos com desfecho favorável (9,95 vs. $16,82 \mu \mathrm{g} / \mathrm{mL}, \mathrm{p}=0,156)$. O fato de uma grande parcela dos pacientes apresentarem piora clínica durante o tratamento com o antibiótico poderia ser relacionado ao surgimento de cepas de hVISA, que estão associadas a níveis séricos abaixo de $10 \mu \mathrm{g} / \mathrm{mL}$ (CASAPAO et al., 2013).

Com relação ao desfecho final, não se verificou diferença entre níveis séricos de vancomicina e mortalidade dos pacientes (Tabela 1). Entre 27 pacientes, somente 16 tiveram confirmação cultural de infecção, sendo que, destes, apenas 9 tiveram infecções ocasionadas por bactérias gram-positivas. Considerando que poucos tratamentos com vancomicina $(33,3 \%, \mathrm{n}=9)$ foram realizados mediante confirmação de infecção por bactérias gram- 
positivas, não foi possível perceber a real influência da concentração sérica do antibiótico nos desfechos clínicos em crianças e neonatos.

\section{CONCLUSÃO}

Através deste estudo podemos concluir que a mensuração dos níveis séricos de vancomicina é de grande importância, uma vez que auxilia na prevenção de toxicidade e no ajuste da prescrição a fim de obter uma concentração terapêutica adequada e evitar o surgimento de cepas resistentes. $\mathrm{O}$ acompanhamento e a monitorização dos pacientes em uso do antibiótico torna-se importante, sendo que a maioria dos pacientes, independente de sexo e de idade, não consegue atingir valores séricos adequados de vancomicina.

\section{REFERÊNCIAS}

ALMEIDA, R. Vancomicina: avaliação do uso em pacientes internados na unidade de terapia intensiva. Dissertação (Mestrado em Ciências Médicas) - Programa de Pós-Graduação em Medicina, UFRGS, 2011.

ALVES, M. L. P.; MELO, G. A. N.; YAMADA, S. S.; NISHIYAMA, P. Therapeutic monitoring of vancomycin. Acta Scientiarum. Health Sciences, v. 34, n. 2, p. 199-204, 2012. Disponível em: < http://dx.doi.org/10.4025/actascihealthsci.v34i2.10617 >.

ASKARI, E.; TABATABAI, S. M.; ARIANPOOR, A.; NASAB, M. N. VanA-positive vancomycin-resistant staphylococcus aureus: Systematic search and review of reported cases. Infectious Diseases in Clinical Practice, v. 21, n. 2, p. 91-93, 2013.

AVENT, M. L.; VASKA, V. L.; ROGERS, B. A.; CHENG, A. C.; VAN HAL, S. J.; HOLMES, N. E.; HOWDEN, B. P.; PATERSON, D. L. Vancomycin therapeutics and monitoring: a contemporary approach. Internal Medicine Journal, v. 43, n. 2, p. 110-119, 2013.

BOOIJINK, C. C.; EL-AIDY, S.; RAJILIC-STOJANOVIC, M.; HEILIG, H. G.; TROOST, F. J.; SMIDT, H.; KLEEREBEZEM, M.; DE VOS, W. M.; ZOETENDAL, E. G. High temporal and inter-individual variation detected in the human ileal microbiota. Environ Microbiol, v. 12, n. 12, p. 3213-3227, 2010.

BROME, L.; SO, T. An Evaluation of Initial Vancomycin Dosing in Infants, Children, and Adolescents. International Journal of Pediatrics, v. 2011, 2011. Disponível em: <http://dx.doi.org/10.1155/2011/470364 >. 
BROWN, J.; BROWN, K. A.; FORREST, A. Outcomes and costs associated with a history of vancomycin exposure in patients with MRSA-related complicated bacteremia and infective endocarditis. Clin Ther, v. 33, n. 10, p. 1475-1482, 2011.

CALFEE, D. P. Methicillin-resistant Staphylococcus aureus and vancomycin-resistant enterococci, and other Gram-positives in healthcare. Curr Opin Infect Dis, v. 25, n. 4, p. 385394, 2012.

CASAPAO, A. M.; LEONARD, S. N.; DAVIS, S. L.; LODISE, T. P.; PATEL, N.; GOFF, D. A.; LAPLANTE, K. L.; POTOSKI, B. A.; RYBAK, M. J. Clinical Outcomes in Patients with Heterogeneous Vancomycin-Intermediate Staphylococcus aureus Bloodstream Infection. Antimicrobial Agents and Chemotherapy, v. 57, n. 9, p. 4252-4259, 2013.

CDC- Centers for Disease Control and Prevention. Staphylococcus aureus resistant to vancomycin--United States, 2002. MMWR Morb Mortal Wkly Rep, v. 51, n. 26, p. 565-567, 2002.

CLSI. Performance Standards for Antimicrobial Susceptibility Testing; Twenty-second Informational Supplement. CLSI document M100-S23. Wayne: Clinical and Laboratory Standards Institute, 2013.

DE WAELE, J. J.; DANNEELS, I.; DEPUYDT, P.; DECRUYENAERE, J.; BOURGEOIS, M.; HOSTE, E. Factors associated with inadequate early vancomycin levels in critically ill patients treated with continuous infusion. International Journal of Antimicrobial Agents, v. 41, n. 5, p. 434-438, 2013.

DELEO, F. R.; OTTO, M.; KREISWIRTH, B. N.; CHAMBERS, H. F. Communityassociated meticillin-resistant Staphylococcus aureus. Lancet, v. 375, n. 9725, p. 1557-1568, 2010.

ELYASI, S.; KHALILI, H.; DASHTI-KHAVIDAKI, S.; MOHAMMADPOUR, A. Vancomycin-induced nephrotoxicity: mechanism, incidence, risk factors and special populations. A literature review. European Journal of Clinical Pharmacology, v. 68, n. 9, p. 1243-1255, 2012.

GORWITZ, R. J.; KRUSZON-MORAN, D.; MCALLISTER, S. K.; MCQUILLAN, G.; MCDOUGAL, L. K.; FOSHEIM, G. E.; JENSEN, B. J.; KILLGORE, G.; TENOVER, F. C.; KUEHNERT, M. J. Changes in the prevalence of nasal colonization with Staphylococcus aureus in the United States, 2001-2004. J Infect Dis, v. 197, n. 9, p. 1226-1234, 2008.

GRACE, E.; GOODBAR, N. H.; FOUSHEE, J. A. Optimizing Vancomycin Dosing in Obese and Morbidly obese Patients with MRSA Infections. Advances in Pharmacoepidemiology \& Drug Safety, S1:003, 2012.

HIRAMATSU, K. Vancomycin-resistant Staphylococcus aureus: a new model of antibiotic resistance. The Lancet Infectious Diseases, v. 1, n. 3, p. 147-155, 2001.

HIRAMATSU, K.; HANAKI, H.; INO, T.; YABUTA, K.; OGURI, T.; TENOVER, F. C. Methicillin-resistant Staphylococcus aureus clinical strain with reduced vancomycin susceptibility. Journal of Antimicrobial Chemotherapy, v. 40, n. 1, p. 135-136, 1997. 
HOLLAND, T. L.; FOWLER, V. G. Vancomycin Minimum Inhibitory Concentration and Outcome in Patients With Staphylococcus aureus Bacteremia: Pearl or Pellet? Journal of Infectious Diseases, v. 204, n. 3, p. 329-331, 2011.

HOWDEN, B. P.; DAVIES, J. K.; JOHNSON, P. D. R.; STINEAR, T. P.; GRAYSON, M. L. Reduced Vancomycin Susceptibility in Staphylococcus aureus, Including VancomycinIntermediate and Heterogeneous Vancomycin-Intermediate Strains: Resistance Mechanisms, Laboratory Detection, and Clinical Implications. Clinical Microbiology Reviews, v. 23, n. 1, p. 99-139, 2010.

JAMES, C. W.; GURK-TURNER, C. Recommendations for monitoring serum vancomycin concentrations. Proc (Bayl Univ Med Cent), v. 14, n. 2, p. 189-190, 2001.

KIM, D. I.; IM, M. S.; CHOI, J. H.; LEE, J.; CHOI, E. H.; LEE, H. J. Therapeutic monitoring of vancomycin according to initial dosing regimen in pediatric patients. Korean J Pediatr, v. 53, n. 12, p. 1000-1005, 2010.

KOLLEF, M. H. Limitations of vancomycin in the management of resistant staphylococcal infections. Clin Infect Dis, v. 45 Suppl 3, n., p. S191-195, 2007.

KULLAR, R.; DAVIS, S. L.; LEVINE, D. P.; RYBAK, M. J. Impact of vancomycin exposure on outcomes in patients with methicillin-resistant Staphylococcus aureus bacteremia: support for consensus guidelines suggested targets. Clin Infect Dis, v. 52, n. 8, p. 975-981, 2011.

LECLERCQ, R.; DERLOT, E.; DUVAL, J.; COURVALIN, P. Plasmid-mediated resistance to vancomycin and teicoplanin in Enterococcus faecium. $N$ Engl J Med, v. 319, p. 157-161, 1988.

LEVINE, D. P. Vancomycin: A History. Clinical Infectious Diseases, v. 42, n. Supplement 1, p. S5-S12, 2006.

LIU, C.; BAYER, A.; COSGROVE, S. E.; DAUM, R. S.; FRIDKIN, S. K.; GORWITZ, R. J.; KAPLAN, S. L.; KARCHMER, A. W.; LEVINE, D. P.; MURRAY, B. E.; RYBAK, M. J.; TALAN, D. A.; CHAMBERS, H. F. Clinical Practice Guidelines by the Infectious Diseases Society of America for the Treatment of Methicillin-Resistant Staphylococcus aureus Infections in Adults and Children. Clinical Infectious Diseases, v. 52, n. 3, p. e18-e55, 2011.

LODISE, T. P.; GRAVES, J.; EVANS, A.; GRAFFUNDER, E.; HELMECKE, M.; LOMAESTRO, B. M.; STELLRECHT, K. Relationship between vancomycin MIC and failure among patients with methicillin-resistant Staphylococcus aureus bacteremia treated with vancomycin. Antimicrob Agents Chemother, v. 52, n. 9, p. 3315-3320, 2008.

NULENS, E.; GOULD, I.; MACKENZIE, F.; DEPLANO, A.; COOKSON, B.; ALP, E.; BOUZA, E.; VOSS, A. Staphylococcus aureus carriage among participants at the 13th European Congress of Clinical Microbiology and Infectious Diseases. Eur J Clin Microbiol Infect Dis, v. 24, p. 145-148, 2005.

OLIVEIRA, G. A. M. D.; DELL'AQUILA, A. M. M. D.; MASIERO, R. L. P.; LEVY, C. E. P.; GOMES, M. S. P.; CUI, L. P.; HIRAMATSU, K. P.; MAMIZUKA, E. M. P. Isolation in 
Brazil of Nosocomial Staphylococcus aureus with Reduced Susceptibility to Vancomycin. Infection Control and Hospital Epidemiology, v. 22, n. 7, p. 443-448, 2001.

PEA, F.; FURLANUT, M.; NEGRI, C.; PAVAN, F.; CRAPIS, M.; CRISTINI, F.; VIALE, P. Prospectively Validated Dosing Nomograms for Maximizing the Pharmacodynamics of Vancomycin Administered by Continuous Infusion in Critically Ill Patients. Antimicrobial Agents and Chemotherapy, v. 53, n. 5, p. 1863-1867, 2009.

PEACOCK, S. J.; DE SILVA, I.; LOWY, F. D. What determines nasal carriage of Staphylococcus aureus? Trends Microbiol, v. 9, n. 12, p. 605-610, 2001.

PÉRICHON, B.; COURVALIN, P. Heterologous Expression of the Enterococcal vanA Operon in Methicillin-Resistant Staphylococcus aureus. Antimicrobial Agents and Chemotherapy, v. 48, n. 11, p. 4281-4285, 2004.

PRICE, J.; BAKER, G.; HEATH, I.; WALKER-BONE, K.; CUBBON, M.; CURTIS, S.; ENRIGHT, M. C.; LINDSAY, J.; PAUL, J.; LLEWELYN, M. Clinical and Microbiological Determinants of Outcome in Staphylococcus aureus Bacteraemia. Int J Microbiol, v. 2010, n., p. $654858,2010$.

REIS, A. G.; GRISI, S. J. Monitorization of blood levels of vancomycin in children with multi-resistant bacterial infections. J Pediatr (Rio J), v. 72, n. 4, p. 225-229, 1996.

ROSENTHAL, V. D.; BIJIE, H.; MAKI, D. G.; MEHTA, Y.; APISARNTHANARAK, A.; MEDEIROS, E. A.; LEBLEBICIOGLU, H.; FISHER, D.; ÁLVAREZ-MORENO, C.; KHADER, I. A.; DEL ROCÍO GONZÁLEZ MARTÍNEZ, M.; CUELLAR, L. E.; NAVOANG, J. A.; ABOUQAL, R.; GUANCHE GARCELL, H.; MITREV, Z.; PIREZ GARCÍA, M. C.; HAMDI, A.; DUEÑAS, L.; CANCEL, E.; GURSKIS, V.; RASSLAN, O.; AHMED, A.; KANJ, S. S.; UGALDE, O. C.; MAPP, T.; RAKA, L.; YUET MENG, C.; THU, L. T. A.; GHAZAL, S.; GIKAS, A.; NARVÁEZ, L. P.; MEJÍA, N.; HADJIEVA, N.; GAMAR ELANBYA, M. O.; GUZMÁN SIRITT, M. E.; JAYATILLEKE, K. International Nosocomial Infection Control Consortium (INICC) report, data summary of 36 countries, for 2004-2009. American Journal of Infection Control, v. 40, n. 5, p. 396-407, 2012.

RYBAK, M.; LOMAESTRO, B.; ROTSCHAFER, J. C.; MOELLERING, R., JR.; CRAIG, W.; BILLETER, M.; DALOVISIO, J. R.; LEVINE, D. P. Therapeutic monitoring of vancomycin in adult patients: a consensus review of the American Society of Health-System Pharmacists, the Infectious Diseases Society of America, and the Society of Infectious Diseases Pharmacists. Am J Health Syst Pharm, v. 66, n. 1, p. 82-98, 2009.

SIEVERT, D. M. P.; RICKS, P. P.; EDWARDS, J. R. M. S.; SCHNEIDER, A. M. P. H.; PATEL, J. P.; SRINIVASAN, A. M. D.; KALLEN, A. M. D.; LIMBAGO, B. P.; FRIDKIN, S. M. D. Antimicrobial-Resistant Pathogens Associated with Healthcare-Associated Infections: Summary of Data Reported to the National Healthcare Safety Network at the Centers for Disease Control and Prevention, 2009-2010. Infection Control and Hospital Epidemiology, v. 34, n. 1, p. 1-14, 2013.

SORIANO, A.; MARCO, F.; MARTINEZ, J. A.; PISOS, E.; ALMELA, M.; DIMOVA, V. P.; ALAMO, D.; ORTEGA, M.; LOPEZ, J.; MENSA, J. Influence of vancomycin minimum inhibitory concentration on the treatment of methicillin-resistant Staphylococcus aureus bacteremia. Clin Infect Dis, v. 46, n. 2, p. 193-200, 2008. 
UEKOTTER, A.; PETERS, G.; BECKER, K. Is there any rationale for treatment of Staphylococcus aureus infections with antimicrobials that are determined to be ineffective in vitro? Clin Microbiol Infect, v. 17, n. 8, p. 1142-1147, 2011.

UTTLEY, A.H.; COLLINS, C. H.; NAIDOO, J.; GEORGE, R. C. Vancomycin-resistant enterococci. Lancet, v. 1, p. 57-58, 1988.

VAN HAL, S. J.; PATERSON, D. L. Systematic Review and Meta-Analysis of the Significance of Heterogeneous Vancomycin-Intermediate Staphylococcus aureus Isolates. Antimicrobial Agents and Chemotherapy, v. 55, n. 1, p. 405-410, 2011.

VAN HAL, S. J.; PATERSON, D. L.; LODISE, T. P. Systematic Review and Meta-Analysis of Vancomycin-Induced Nephrotoxicity Associated with Dosing Schedules That Maintain Troughs between 15 and 20 Milligrams per Liter. Antimicrobial Agents and Chemotherapy, v. 57, n. 2, p. 734-744, 2013.

VANDECASTEELE, S. J.; DE BACQUER, D.; DE VRIESE, A. S. Implementation of a Dose Calculator for Vancomycin to Achieve Target Trough Levels of $15-20 \mu \mathrm{g} / \mathrm{mL}$ in Persons Undergoing Hemodialysis. Clinical Infectious Diseases, v. 53, n. 2, p. 124-129, 2011.

WILLEMS, R. J.; HANAGE, W. P.; BESSEN, D. E.; FEIL, E. J. Population biology of Gram-positive pathogens: high-risk clones for dissemination of antibiotic resistance. FEMS Microbiol Rev, v. 35, n. 5, p. 872-900, 2011.

YE, Z. K.; TANG, H. L.; ZHAI, S. D. Benefits of Therapeutic Drug Monitoring of Vancomycin: A Systematic Review and Meta-Analysis. PLoS ONE, v. 8, n. 10, p. E77169, 2013.

YOON, Y. K.; KIM, J. Y.; PARK, D. W.; SOHN, J. W.; KIM, M. J. Predictors of persistent methicillin-resistant Staphylococcus aureus bacteraemia in patients treated with vancomycin. J Antimicrob Chemother, v. 65, n. 5, p. 1015-1018, 2010. 